\title{
Telomere in colorectal cancer associated with distant metastases and predicted a poor prognosis
}

\author{
Xiaorong Ye, Jianjiong Li, Chenjun Song, Wenbin Chen \\ Department of Colorectal Surgery, The First Affiliated Hospital, Zhejiang University School of Medicine, Hangzhou, China \\ Contributions: (I) Conception and design: W Chen; (II) Administrative support: W Chen; (III) Provision of study materials or patients: W Chen; (IV) \\ Collection and assembly of data: X Ye, J Li, C Song; (V) Data analysis and interpretation: X Ye, J Li, W Chen; (VI) Manuscript writing: All authors; \\ (VII) Final approval of manuscript: All authors. \\ Correspondence to: Wenbin Chen, MD. Department of Colorectal Surgery, the First Affiliated Hospital, Zhejiang University School of Medicine, No. \\ 79, Qingchun Road, 310003 Hangzhou, China. Email: wenbinchen@zju.edu.cn.
}

Background: Telomere is essential for chromosomal stability and its length has been proven to be related to prognosis in many malignant tumors. This study aims to investigate the relevance of telomere length with clinical and pathologic features and its prognostic value in colorectal cancer (CRC).

Methods: Telomere status of CRC and adenoma cells were measured by telomere-specific quantitative fluorescent in situ hybridization (Q-FISH). The relative telomere length (RTL) was calculated as the mean telomere fluorescent intensity units (TFUs) in carcinoma cell divided by the TFU in cancer-associated fibroblast cell (CAF).

Results: One hundred CRC patients, who were received surgery treatment during 2013 to 2014 and fifty-seven patients who underwent the examination of colonoscope and were confirmed as adenoma were enrolled. TFUs of carcinoma cell and CAF were statistically significantly lower than in adjacent mucosa cell $(\mathrm{P}=0.0079)$. Although there was no difference between the three kinds of adenoma cells $(\mathrm{P}=0.5457)$, TFU in adenoma cells was significantly lower than in CAF $(\mathrm{P}<0.0001)$ and independent with age. TFU and the RTL were statistically significantly lower in adenoma cells than in carcinoma (all $\mathrm{P}<0.0001$ ). TFU of carcinoma cell in distant metastases patients were significantly lower than that without distant metastases patients $(\mathrm{P}=0.002)$. When cut by the median value of TFU of carcinoma cell and RTL, patients with a lower TFU or RTL had statistically significantly poorer overall survival (OS) (P=0.0027, HR: 4.6, 95\% CI: 1.9-11.0; $\mathrm{P}=0.0163$, HR: 2.95, 95\% CI: 1.22-7.12) and disease-free survival (DFS) (P=0.0057, HR: 3.14, 95\% CI: 1.40-7.06; P=0.0271, HR: 2.49, 95\% CI: 1.11-5.59, respectively) than those patients with higher TFU or RTL. On multivariate analysis, the TFU of carcinoma cell was proved to be an independent prognostic value both for OS and DFS (P=0.0005, HR: 4.975, 95\% CI: 1.616-15.385; P=0.007, HR: 3.57, 95\% CI: $1.410-$ 9.010).

Conclusions: The length of telomere in carcinoma and adenoma cells were consistently shorter and the telomere changes were early carcinogenesis event, even the epithelial cells were morphologically not malignant. The length of telomere was associated with tumor metastases and prognosis, suggesting telomere probably was an important cue of the biological behavior of CRC.

Keywords: Colorectal cancer (CRC); telomere; prognosis; quantitative fluorescent in situ hybridization (Q-FISH)

Submitted Nov 29, 2020. Accepted for publication Apr 13, 2021.

doi: $10.21037 /$ tcr-20-3341

View this article at: https://dx.doi.org/10.21037/tcr-20-3341 


\section{Introduction}

Colorectal cancer (CRC) is the third most common cancer and the second cause of cancer death worldwide and 883,200 deaths are estimated to have occurred in 2018 (1). Great progress has made, such as increased awareness and early detection, improved treatments on surgery and chemotherapy, which have all contributed to prolonged survival though, CRC is still the most common cause of cancer-related deaths. CRC involves multi-step transformation of normal colon tissue to precancerous polyps or adenomas and finally to a malignant neoplasm. Not all polyps or adenomas are evolved into CRC, but for most colorectal malignancies, clinical and epidemiological evidences clearly indicate that they are the precursor lesions (2). In the progression of adenoma-carcinoma transition, different genetic and epigenetic events are involved (3).

Telomere is repetitive DNA sequences (TTAGGG) located at the end of chromosomes and its main functions is stabilizing chromosomes by protecting them from end-toend fusion and DNA degradation $(4,5)$. Telomere undergoes a progressive shortening during each cell-replication cycle because of incomplete DNA replication of a lagging strand (so called as the end-replication problems) and telomere shortening induces somatic cells to undergo senescence and apoptosis (4). So, in this sense, telomere may act as a tumorsuppressing mechanism, preventing cells from uncontrolled division. However, continuous erosion of telomere may impair its function in protecting chromosome ends, leading to chromosomal instability, a key event in the initiation of carcinogenesis and cancer progression (6-8).

As we known, chromosomal instability and microsatellite instability were considered as the two main pathways of CRC carcinogenesis (9). Telomere shortening is identified as an early event in CRC carcinogenesis. Of note, most (approximately $85 \%$ ) of CRCs are associated with chromosomal instability and telomere dysfunction is considered as a fundamental player in this process (10). Telomere shortening was frequently found in adenomacarcinoma transition and telomere dysfunction initiated cancer formation by induction of chromosomal instability (11-13). While most studies identified telomere shortening as a critical initial event in carcinogenesis, the role of telomere length in cancer cells as a marker of disease progression was controversial (14). In fact, no agreement concerning the role of telomere length as a marker of disease progression has been reached in CRC. A few researches indicated that telomere length in cancer tissue was significantly longer in the late stage of colorectal tumors $(15,16)$, other studies had not indicated any correlation between telomere length and stage (17-19). In one study, shorter telomere length (TL) was observed in tumors with earlier stage, but not in those with advanced stage (20). As for a prognostic role, telomere length hadn't been confirmed either. Only in some of these studies indicated longer telomeres were associated with poor clinical outcome $(16,19,21)$.

In tumor microenvironment, cancer-associated fibroblast cell (CAF) constituted the majority cell type and was considered as a crucial role in CRC, such as tumor growth, progression, metastasis, angiogenesis and immune responses (22). CAF was originally activated by tumor cells from a subpopulation of fibroblasts and was consider as prognostic biomarkers in cancers (23). In prostate cancer cell, shorter telomere length in cancer-associated stromal cell (fibroblasts and smooth muscle) were prognostic marker for progress to metastasis and die of their prostate cancer (24).

Considering the relevance of telomere in carcinogenesis and its role as prognostic biomarkers, our main aim in this work was to highlight the changes of telomere in difference kinds of adenomas and to clarify the relevance between the clinical and pathologic features and its prognostic value in CRC. With this objective, we evaluated the telomere status of CRC and adenoma by telomere-specific quantitative fluorescent in situ hybridization (Q-FISH) with which could provide single cell resolution of telomere length while maintaining tissue architecture. We present the following article in accordance with the REMARK reporting checklist (available at https://dx.doi.org/10.21037/tcr-20-3341).

\section{Methods}

\section{Patients}

One hundred patients with CRC, who were received surgery treatment at the Department of Colorectal Surgery, the first affiliated hospital, ZheJiang University School of Medicine during 2015 to 2016, were enrolled in the present study. Biopsy tissues which were obtained from fifty-seven patients who underwent the examination of colonoscope during 2013 and were confirmed as adenoma were also used. Form the reports of pathological diagnosis, sixteen were serrated adenoma, nineteen were tubular adenoma and twenty-two were villous adenoma. Patients received 
(neo)adjuvant radiotherapy or chemotherapy before operation were excluded. The study was conducted in accordance with the Declaration of Helsinki (as revised in 2013). The study was approved by the ethics committee of the first affiliated hospital, ZheJiang University School of Medicine (No.2020-656) and informed consent was taken from all the patients.

\section{Q-FISH}

Before Q-FISH, the pathological diagnosis was confirmed by a pathologist under a microscope though the standard H.E sections. Paraffin-embedded, 4- $\mu$ m-thick sections adjacent to the tissue used for H.E. sections were obtained. After dewaxing in xylene hydration in alcohol series $(100 \% ; 90 \% ; 70 \%)$, sections were boiled in microwave oven for 10 minutes in citrate-buffer $(0.01 \mathrm{M}, \mathrm{H}$ : 6.0 Rinsed in phosphate buffer saline (PBS), sections were then incubated in preheated pepsin solution for 5 minutes $\left(37^{\circ} \mathrm{C}\right)$. After dehydrated the slides following the alcohol series $(70 \% ; 90 \% ; 100 \%)$ and air dried the sections about 5 minutes until all alcohol was gone, $15 \mu \mathrm{L}$ hybridisation $\operatorname{mix}[10 \mathrm{mM}$ Tris, $2.14 \mathrm{mM} \mathrm{MgCl}$; $0.5 \%$ Blocking reagent (Roche), $0.1 \mathrm{nM} \mathrm{Cy} 3$ conjugated telomere peptide nucleic acid probe (sequence: Cy3-OO-(CCCTAA) catalog\#F1002, Panagene Inc., Korea] were applied and covered with a coverslip. For hybridization, slides were denatured at $80{ }^{\circ} \mathrm{C}$ for $3 \mathrm{~min}$ and incubated in a humid chamber for two hours. At last, the sides were rinsed in wash buffer $(0.1 \%$ bovine serum albumin and $10 \mathrm{mMTris,}$ $70 \%$ Formamide (Sigma-aldrich, catalog\#34724-1L-R), TBS-T (TBS plus $0.1 \%$ tween-100) and PBS, and applied about 30pl mounting solution Antifade+4',6-diamidino-2phenylindole (DAPI) (catalog\#H-1200, Vector) and covered with a coverslip.

During each experiment, a slide of normal colon mucosa was used as internal control. Pictures were taken under a fluorescence microscope $(100 \times$ objective lens and $10 \times$ ocular lens, Olympus BX51, Japan) with the software of NIS-Elements. Both Cy3 (telomere signal) and DAPI (nuclear stain signal) images were captured under the same microscope field. The exposure time was held constant at $150 \mathrm{~ms}$ for all $\mathrm{Cy} 3$ images to keep the signals within the linear range of the camera and enable comparison of telomere signal. At least ten pictures form different sites of the sample were taken. Telomere length were measured in carcinoma cells and CAF cells. At least 50 carcinoma or adenoma cells, CAF cells of the total telomere fluorescent intensity units (TFUs) were quantitatively measured by the software of TFL-TeloV2.

\section{Statistical analysis}

Investigators were blinded to the pathological information of any patient at the time of analysis. The relative telomere length (RTL) was calculated as the TFU in carcinoma cell or adjacent mucosa divided by the TFU in CAF cell. TFUs and RTL were expressed as median (interquartile range). An interquartile range was defined as the distribution of TFU values between 25 th and 75 th percentiles. Statistical analyses were conducted on natural data by using MannWhitney tests. Differences between TFU in more than two parameters were analyzed using non-parametrical ANOVA (Kruskal-Wallis test). The relationship between the patient age at diagnosis and TFU values was calculated by Pearson correlation coefficient. Overall survival (OS) was defined as time from study enrolment until death or last follow-up. Disease-free survival (DFS) was defined as time from study enrolment until cancer recurrence or metastases or death without evidence of recurrence or a second primary tumor, or date of last visit. The median values were used as cutoff. Based on the cut-off, CRC patients were stratified into two groups. Survival curves were plotted using KaplanMeier method and log-rank test was used for comparison. Multivariate analysis was performed by multivariate Cox model. Statistical analyses were conducted using Graphpad Prism 8 (GraphPad Software, Inc., La Jolla, CA, USA) and SPSS (version 19.0) software (IBM Corporation, Armonk, NY, USA). P values $<0.05$ were considered statistically significant.

\section{Results}

\section{Clinical characteristics of CRC patients}

The description of the population including fundamental characteristics of CRC and background variables were summarized in Table 1. The median age for the group of CRC patients was 61 years (range, 33-88 years); of those, $45 \%$ were men and $55 \%$ were women. As for the 57 adenoma patients, 16 were serrated adenomas, 19 were tubular adenoma, 22 were villous adenoma. The median age was 56 years (range, 31-65 years), 31 (54\%) were men and $26(46 \%)$ were women.

All patients were subject to a follow-up process, and the examinations including abdomen contrast-enhanced 
Table 1 Clinical and pathologic features of colorectal cancer patients

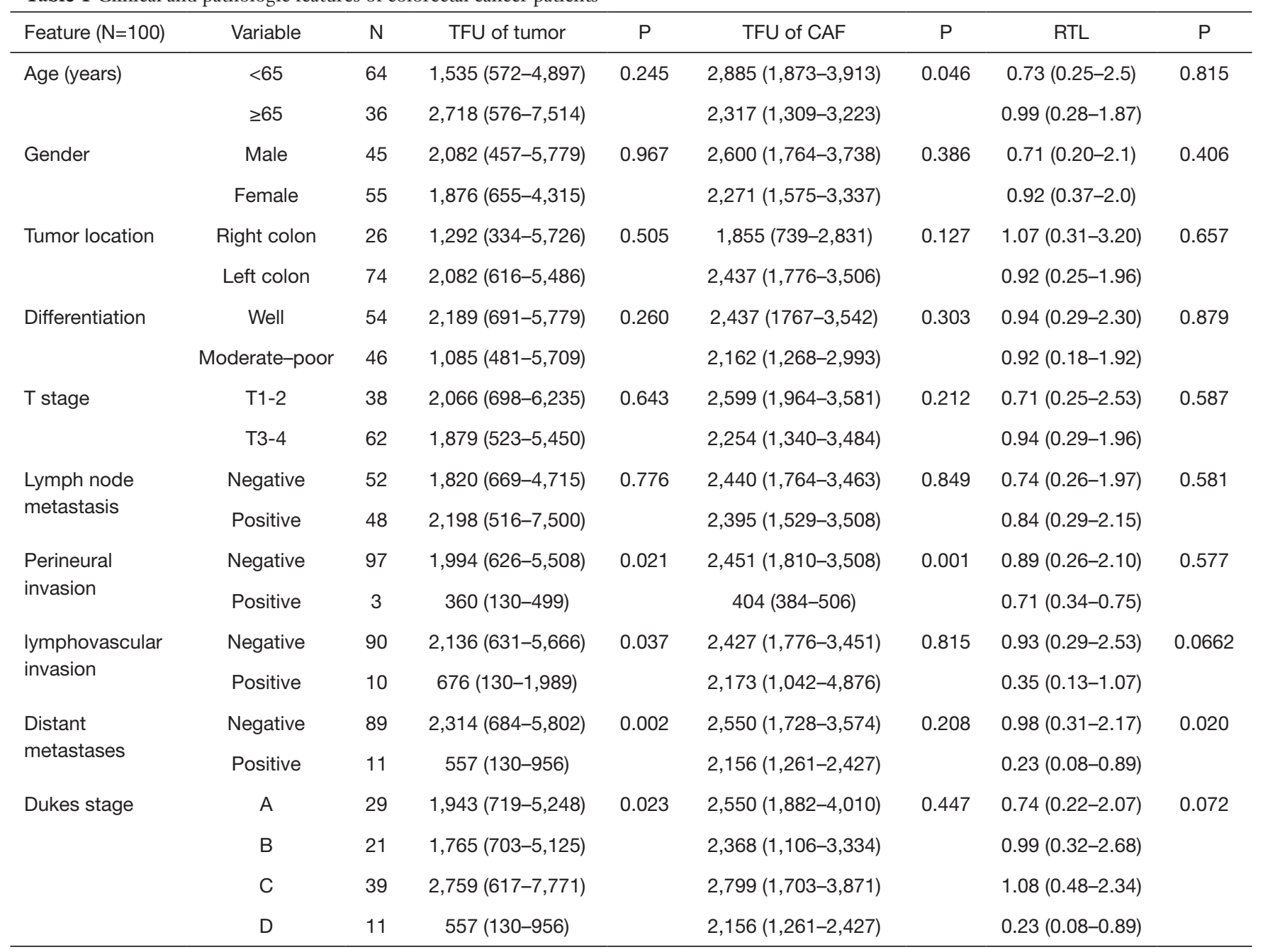

TFU, telomere fluorescent intensity units; CAF, cancer-associated fibroblast cell; RTL, relative telomere length.

computed tomography and colonoscopy were appointed at 1 month after the treatment. The last update of patient follow-up for this study was December 2020 and none was lost to follow up. All the patients were followed up for at least 2 years and the median follow-up time was 46 months. During the follow-up, 20 (20\%) patients were dead and 5 $(5 \%)$ patients were suffered from distant metastasis or local recurrence. The 1 and 3-year OS rates of all the patients were estimated as $97 \%$ and $72 \%$.

\section{TFU in tumor, adenoma and adjacent mucosa cell}

The representative images of telomere fluorescence in situ hybridization in CRC were shown in Figure 1. TFUs of carcinoma cells and CAF were statistically significantly lower than in adjacent mucosa [median (interquartile range): $1,968(572-5,519)$ vs. 3,410 $(1,845-4,719)$ vs. 3,425 $(1,890-4,727), \mathrm{P}=0.0079$, Figure $2 A]$. Although there was no significant difference between age in carcinoma cell and RTL, TFUs in CAF were lower in age $\geq 65$ years $[2,885$ $(1,873-3,913)$ vs. $2,317(1,309-3,223), \mathrm{P}=0.046$, Table 1]. What's more, an inverse relationship between TFU in $\mathrm{CAF}$ and age was recorded $(\mathrm{R}=-0.4476$ and $\mathrm{P}<0.0001, \mathrm{Y}$ $=-50.76^{*} \mathrm{X}+5,825$, Figure $2 B$ ), with a decrease telomere at a rate of $50.76 \mathrm{TFU}$ each year. However, neither TFU nor the RTL in carcinoma cell and in adjacent mucosa, the inverse correlations hadn't been found $(r=0.064, \mathrm{P}=0.5236$; $\mathrm{r}=0.1650, \mathrm{P}=0.1009$ and $\mathrm{r}=-0.019, \mathrm{P}=0.8599 ; \mathrm{r}=-0.043$, $\mathrm{P}=0.6737)$. TFUs in carcinoma and in CAF cells and the RTL did not significantly differ with gender, tumor location 

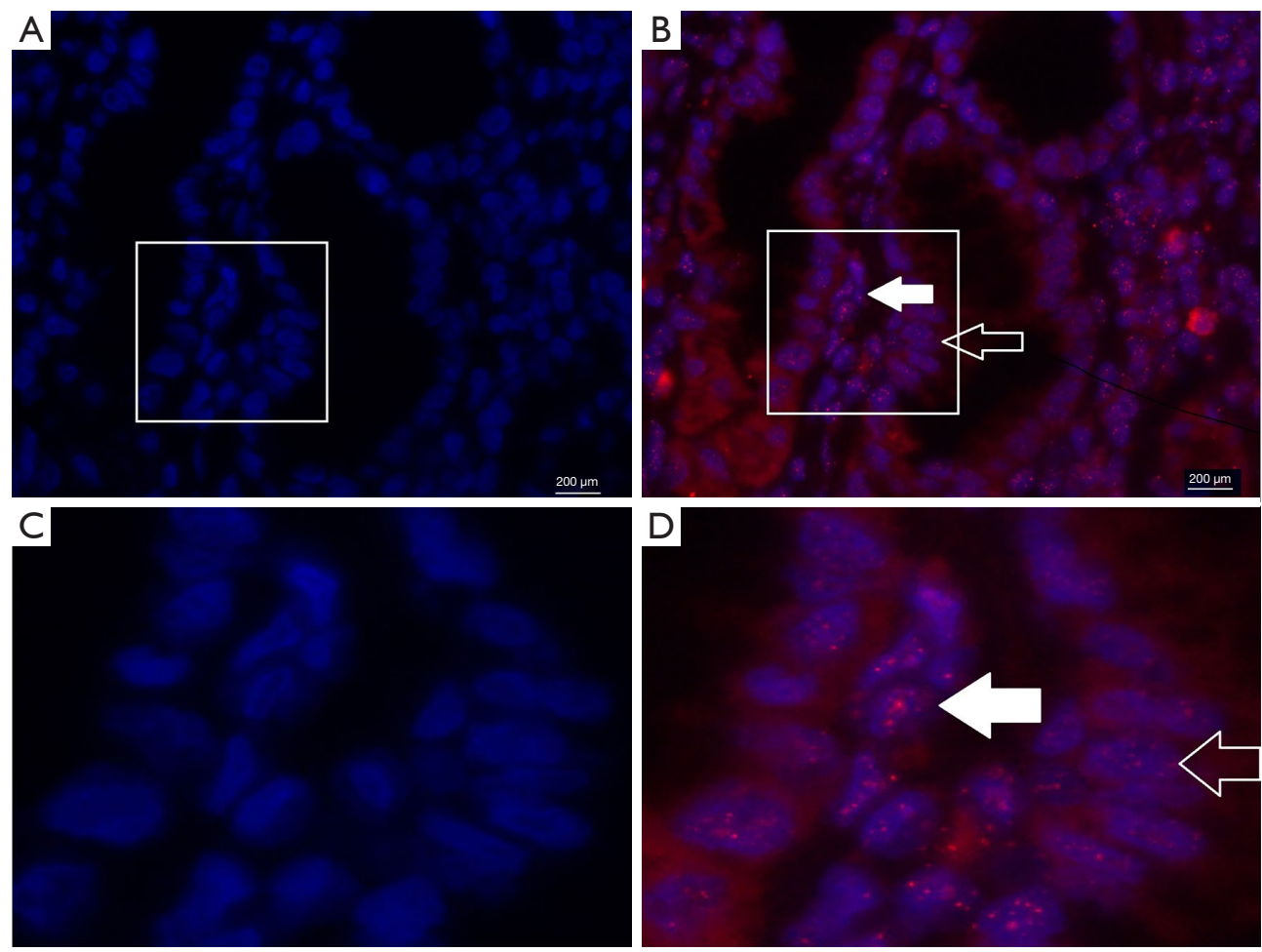

Figure 1 Representative images of telomere fluorescence in situ hybridization in different tissue types. (C) and (D) were the partial enlarged drawing of (A) and (B), respectively. (A) and (C), nuclei were counterstained with DAPI (blue florescence). (B) and (D). Telomere length was reflected by the total red fluorescence intensity in nuclei. In CRC tissue, the CAF cells (filled arrows) show bigger, more numerous and intense red signal red signal than carcinoma cells (open arrows), reflecting longer telomere length in CAF. CAF, cancer-associated fibroblast cell.

and tumor differentiation (Table 1).

We did not find any difference between the three kinds of adenoma cells (serrated, tubular and villous adenoma) [538 (143-2,187) vs. $526(380-2,322)$ vs. $688(153-1,276)$, $\mathrm{P}=0.5457]$. Consistent with carcinoma cells, TFU in adenoma cells was significantly lower than in CAF and in adjacent mucosa $[607(246-1,413)$ vs. $2,178(1,307-$ 2,964) vs. 2,810 (1,916-3,602), $\mathrm{P}<0.0001$, Figure $2 C]$ and also independent with age. TFU in adenoma cells were statistically significantly lower than in carcinoma [607 (246$1,413)$ vs. 1,968 (572-5,519), $\mathrm{P}<0.0001$; Figure 2D]. RTLs in carcinoma, adenoma and adjacent mucosa cell were also compared and RTL in adenoma and adjacent mucosa cell were significantly lower $[0.826(0.26-1.97) v s .0 .362$ (0.11-0.81) vs. 1.353 (1.003-1.588), $\mathrm{P}<0.0001$, respectively. Figure 2E]. To exclude the other influencing factors, TFU of CAF in carcinoma tissue and adenoma tissue were compared, no differences were found $[2,395(1,678-3,497)$ vs. $2,178(1,307-2,964), \mathrm{P}=0.1206]$.

\section{TFU, RTL and tumor progression}

According to $\mathrm{T}$ staging, patients were divided into two groups: T1-2 group and T3-4 group. TFU did not statistically significantly differ between the different tumor $T$ stages $[2,066(698-6,235)$ vs. 1,879 $(523-5,450)$, $\mathrm{P}=0.643]$. TFU did not differ between lymph node metastasis either $[1,820(669-4,715)$ vs. 2,198 (516-7,500), $\mathrm{P}=0.776] .3$ patients were confirmed as perineural invasion and 10 as lymphovascular invasion. TFU of carcinoma cell in perineural invasion and lymphovascular invasion were significantly lower [360 (130-499) vs. 1,994 (626-5,508), $\mathrm{P}=0.021 ; 676(130-1,989)$ vs. $2,136(631-5,666), \mathrm{P}=0.037$, respectively]. TFU of CAF in perineural invasion patients were significantly lower too (404 (384-506) vs. 2,451 $(1,810-3,508), \mathrm{P}=0.001] .11$ patients suffering from distant metastases in this study and TFU of carcinoma cell in distant metastases patients were significantly lower than that without distant metastases patients $[557$ (130-956) vs. 2,314 (684-5,802), $\mathrm{P}=0.002]$. By Dukes stage, 29 belong to 
A

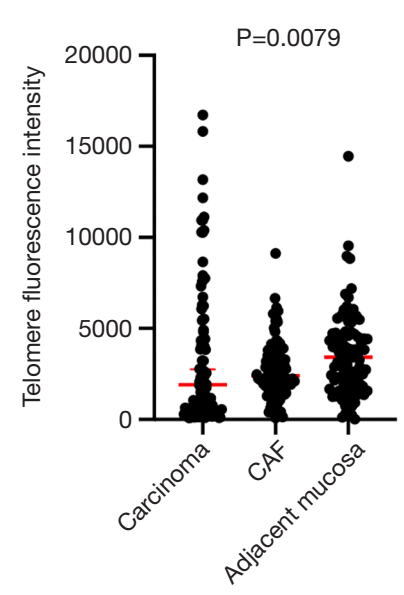

B

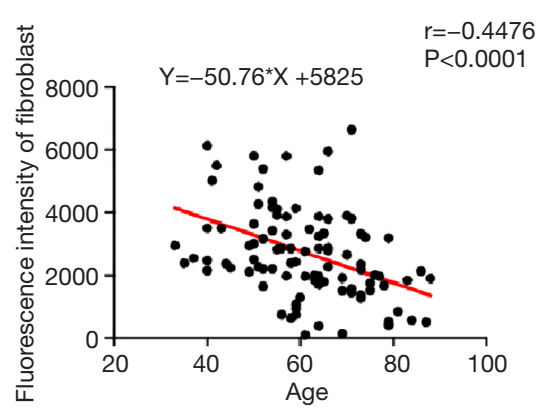

C

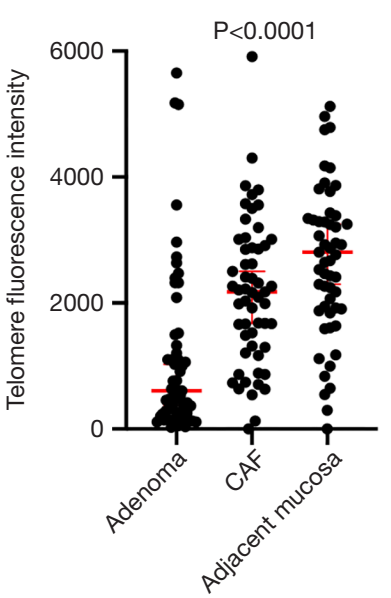

D
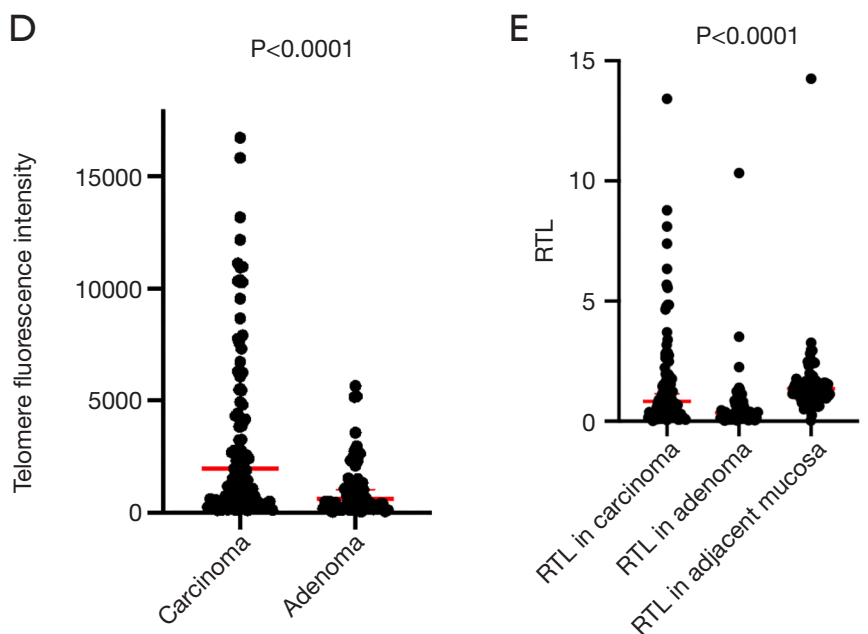

Figure 2 TFU and RTL changes in carcinoma, adenoma and cancer-associated fibroblast (CAF) cells. (A) TFUs in carcinoma cells were statistically significantly lower than in CAF. (B) There was an inverse relationship between TFU in CAF and age, with a decrease telomere at a rate of 50.76 TFUs each year. (C) TFU in adenoma cells was significantly lower than in CAF. (D,E) Both TFU and the RTL were statistically significantly lower in adenoma cells. TFU, telomere fluorescent intensity units; RTL, relative telomere length; CAF, cancerassociated fibroblast cell.

stage A, 21 stage $\mathrm{B}, 39$ stage $\mathrm{C}$ and 11 stage $\mathrm{D}$ and TFU was significantly lower than other stage patients $[1,943$ $(719-5,248)$ vs. $1,765(703-5,125)$ vs. 2,759 (617-7,771) vs. 557 (130-956), for all, $\mathrm{P}=0.023$ and $\mathrm{A}$ and $\mathrm{D}, \mathrm{P}=0.0352$; $\mathrm{B}$ and $\mathrm{D}, \mathrm{P}=0.0417 ; \mathrm{C}$ and $\mathrm{D}, \mathrm{P}=0.0124]$. However, except that patients with distant metastases had significantly lower RTL [0.23 (0.08-0.89) vs. 0.98 (0.31-2.17), $\mathrm{P}=0.020]$, neither TFU in CAF nor the RTL had relevant to tumor progression (Table 1).

\section{Lower TFU and RTL were associated with poor prognosis}

Kaplan-Meier method and the log-rank test was used for comparison of outcomes and the median values of the TFU of carcinoma cell (1909), CAF (2399), adjacent mucosa (3281) and RTL (0.826) were used as cutoff. Patients with a lower TFU or RTL had statistically significantly poorer OS than those patients with higher TFU or RTL $(\mathrm{P}=0.0027$, HR: 4.6, 95\% CI: 1.9-11.0; P=0.0163, HR: 2.95, 95\% CI: 
A

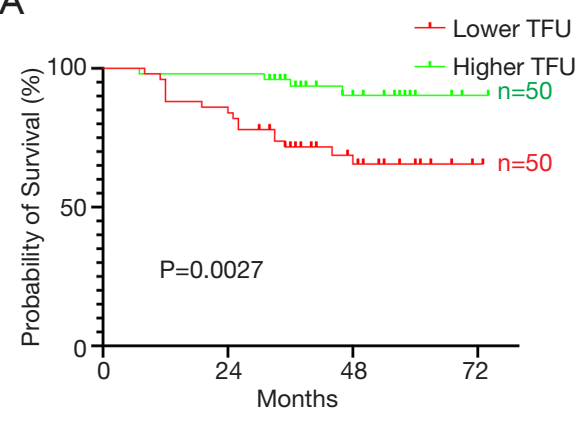

C

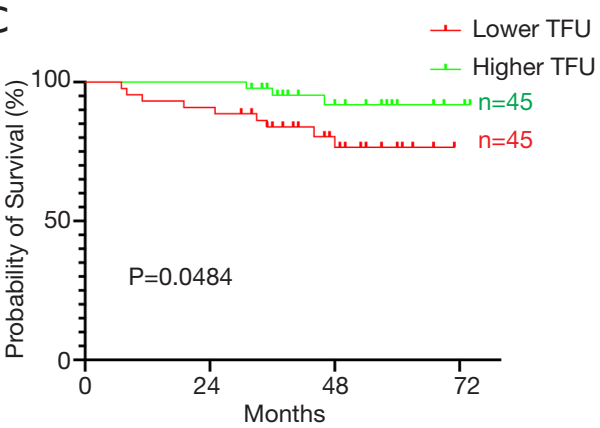

B
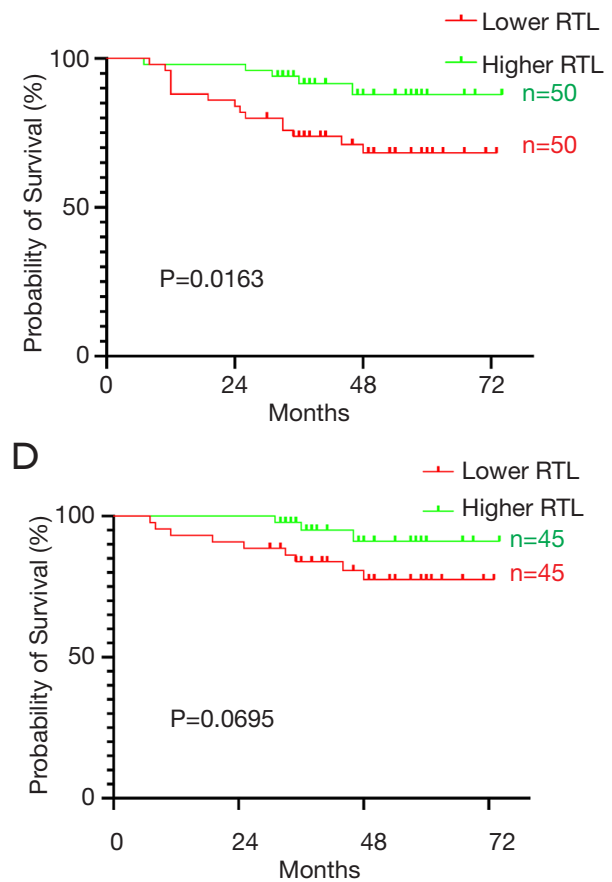

Figure 3 Lower TFU and RTL were associated with OS. (A) Patients with a lower TFU had statistically significantly poorer OS than those patients with higher TFU. (B) Lower RTL patients had statistically significantly poorer OS. (C) After excluded Dukes D stage patients, lower TFU still predicted a poorer OS. (D) Lower RTL was associated with short OS, although the difference was not significant. TFU, telomere fluorescent intensity units; RTL, relative telomere length; OS, overall survival.

1.22-7.12, respectively. Figure $3 A, B)$. Given the Dukes D stage patients had much lower TFU and $72.7 \%(8 / 11)$ were dead during follow up. To test the prognostic value, we excluded Dukes D stage patients and found that only lower TFU still predicted a poorer OS ( $\mathrm{P}=0.0484$, HR: 3.4, 95\% CI: 1.1-10.7; Figure 3C), although the difference in RTL for OS trend to be significant $(\mathrm{P}=0.0695$, HR: 2.86, 95\% CI: 0.92-8.87. Figure 3D). As for DFS, lower TFU or RTL also had a poor prognosis $(\mathrm{P}=0.0057$, HR: $3.14,95 \%$ CI: $1.40-7.06 ; \mathrm{P}=0.0271$, HR: $2.49,95 \%$ CI: $1.11-5.59$, respectively. Figure $4 A, B)$. After excluded Dukes D stage patients, prognosis of patients with lower TFU trend to be poor ( $\mathrm{P}=0.0878$, HR: 2.64, 95\% CI: 0.92-7.52. Figure $4 C)$ but the difference in RTL was not significant $(\mathrm{P}=0.1103$, HR: 2.484, 95\% CI: 0.87-7.08. Figure 4D). However, TFU of $\mathrm{CAF}$ and adjacent mucosa did not show any prognostic value, neither for OS $(\mathrm{P}=0.3011$, HR: $1.592,95 \% \mathrm{CI}$ : .0.66-3.8; $\mathrm{P}=0.8672$, HR: 1.078 , 95\% CI: 0.45-2.6) nor DFS ( $\mathrm{P}=0.1836$, HR: $1.731,95 \%$ CI: 0.77-3.9; $\mathrm{P}=0.3024$, HR: 1.532 , 95\% CI: 0.68-3.45). Other clinicopathological parameters, such as tumor differentiation, $\mathrm{T}$ stage, lymph node metastasis and distant metastases were also associated with prognosis and gender, age and tumor location were not (Table 2,3).

In the next step, TFU of carcinoma cell and RTL, together with tumor differentiation, $\mathrm{T}$ stage, lymph node metastasis and distant metastases were included in multivariate Cox proportional hazards analysis and TFU of carcinoma cell ( $\mathrm{P}=0.0005$, HR: 4.975 , 95\% CI: 1.616-15.39), lymph node metastasis ( $\mathrm{P}=0.045$, HR: 3.139, 95\% CI: 1.026-9.60) and distant metastases ( $\mathrm{P}=0.001$, HR: 5.475, 95\% CI: 2.027-14.792) turn out to be independent prognostic factors for OS (Table 2) and TFU of carcinoma cell ( $\mathrm{P}=0.007$, HR: 3.57, 95\% CI: 1.410-9.010) and distant metastases ( $\mathrm{P}=0.001$, HR: 10.11, 95\% CI: 3.896-26.22) were independent prognostic factors for DFS (Table 3).

\section{Discussion}

In present study, we demonstrated that TFUs in carcinoma and adenoma cells were consistently lower than in CAF and the TFUs in adenoma cell were even lower than in 

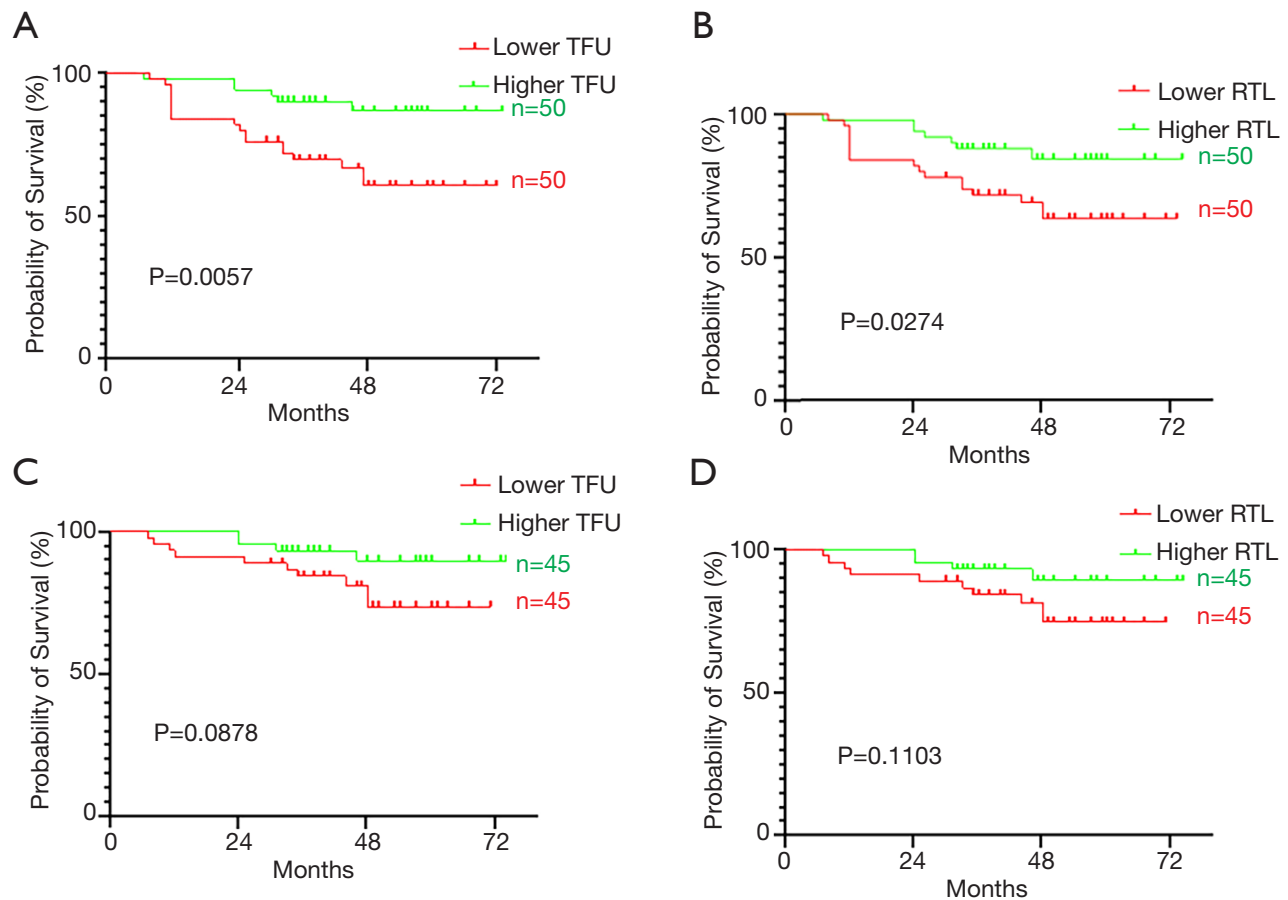

Figure 4 Lower TFU and RTL were associated with DFS. (A) Patients with a lower TFU were associated with short DFS. (B) Lower RTL patients had statistically significantly poorer DFS. (C) After excluded Dukes D stage patients, lower TFU was associated with short DFS, although the difference was not significant. (D) Lower RTL was not associated with DFS when Dukes D stage patients were taken off. TFU, telomere fluorescent intensity units; RTL, relative telomere length; DFS, disease-free survival.

Table 2 Univariate and multivariate cox regression analysis of OS for univariate and multivariate

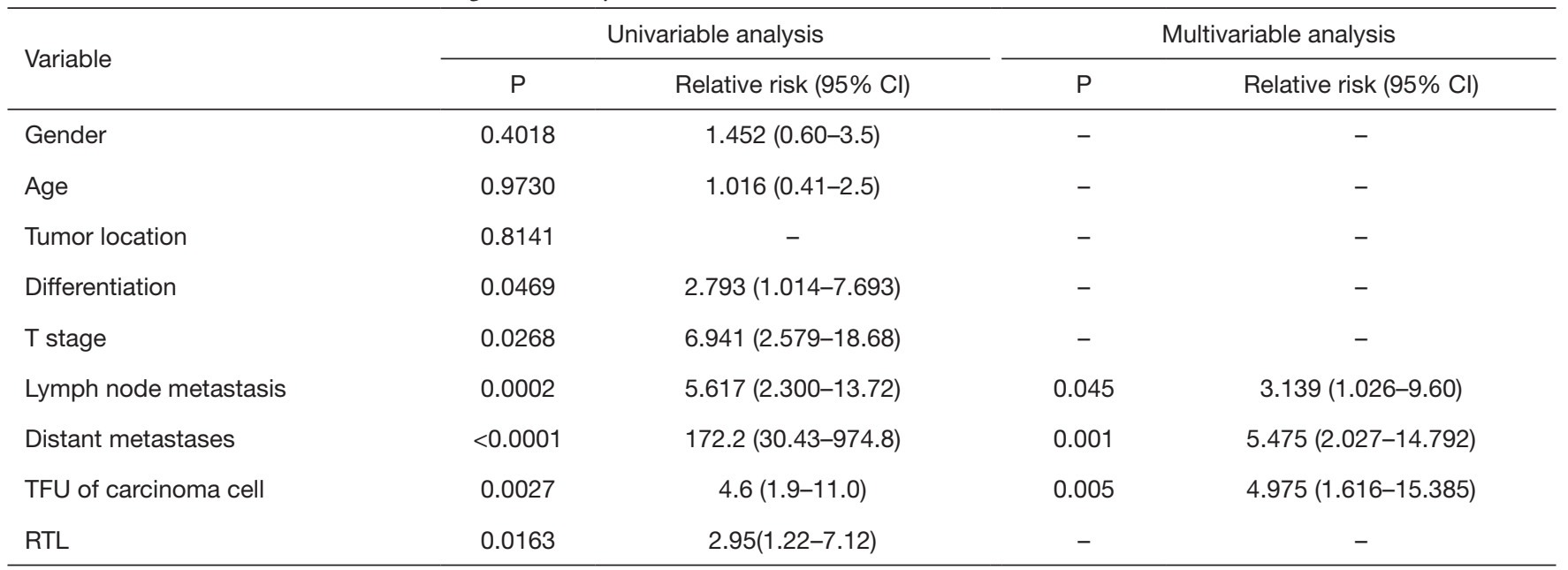

OS, overall survival; RTL, relative telomere length.

carcinoma. This result supported the hypothesis that telomere changes were early carcinogenesis event, even the epithelial cells were morphologically not malignant. TFUs in carcinoma and RTL were associated with tumor metastases and patients who had low TFU or RTL had significantly poor prognosis.

Telomere is progressive shortening because of the endreplication problems and continuous erosion of telomere 
Table 3 Univariate and Multivariate cox regression analysis of DFS for univariate and multivariate

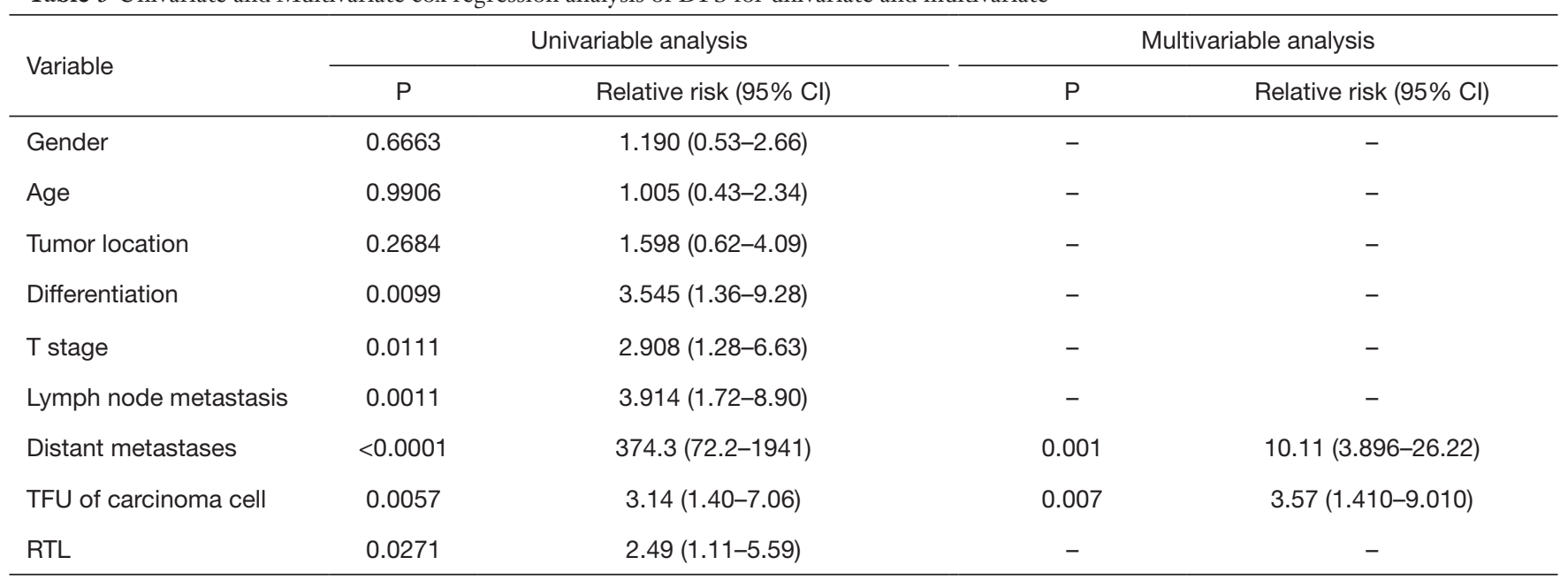

DFS, disease-free survival; RTL, relative telomere length.

may impair its function in protecting chromosome ends, leading to genetic instability (6-8). Chromosomal instability and microsatellite instability are identified as the two main pathways of CRC carcinogenesis $(9,25)$. CRC were thought to arise from the adenoma-carcinoma sequence by sequential accumulation of genetic alterations (26). However, the changes of telomere in the adenoma-carcinoma sequence has not been well established. Consistent with previous reports, it seemed certain that telomere length in CRC was shorter than in normal mucosa (11-13,15-20). Evidences showed telomere length was shorter in low-grade and high-grade dysplasia than in carcinoma (27). Our results shown that telomere in adenomas were shorted than CAF and carcinoma cell, which were consistent with Nirosha Suraweera's observation: adenoma telomere length was significantly shorter than matched normal mucosa, more prevalent tumor telomere shortening than carcinoma patients, indicated that shortened telomeres and telomere maintenance engagement occur early in adenomatous polyp development (28). Clinically, only a small fraction of adenomas actually become malignant, it is hard to predict which adenomas will progress. Bertorelle et al. argued that aggressive polyps had shorter telomeres than carcinoma free adenomas and telomere could distinguish malignant from benign adenomas (10). Another research also observed that different adenoma had its own mechanism: tubular adenomas were associated with mutations of $A P C, K R A S$, and $p 53$, whereas serrated adenomas progressed through microsatellite instability and $B R A F$ mutations (9). However, as for telomere length, tubular and serrated adenoma were found to be similar (11). Our results were the same, the difference of telomere length in different adenoma subtypes was not significant (date not shown), suggesting telomere shorting was common phenomenon in adenoma. Given the intensive cell proliferation with telomere loss and the relatively longer telomere length, mechanisms of telomeric sequences synthesis, such as up-regulation of telomerase, should be involved. Indeed, the trend of growing telomerase activity in the adenoma-carcinoma sequence, as well as the decrease in telomere length has been reported (29). Taken together, our results strongly supported the concept that telomere erosion is a critical initial event in colorectal carcinogenesis. Further studies are required to better understand the role of telomere in the pathological progression in colorectal neoplasia.

CAF constituted the majority cell type in tumor microenvironment and play a crucial role in tumor growth, progression, metastasis, angiogenesis (22). We measured the telomere of CAF in carcinoma and adenoma simultaneously. As mentioned above, telomere was progressively shortened with each successive cell division and therefor associated with aging (4). Our results supported this concept and TFUs in CAF were lower in age $\geq 65$ years, but not in carcinoma cell. What's more, the TFUs in CAF were found to be inversely correlated with age, which was in line with previous studies found in normal mucosa tissue $(16,18,30)$. This negative correlation has also been demonstrated in other malignancies, such as breast cancer (31). To the best of our knowledge, this study is the first to report that telomere changes in $\mathrm{CAF}$ and record the inverse relationship between 
TFU in CAF and age.

The relationship between telomere and CRC progression was not entirely clear. Some investigators identified that telomeres were longer in late stage cases $(16,21)$, others could not confirm the correlation between telomere length and disease stage (17-19,29). Our date showed that TFU of carcinoma cell and RTL were stable in Dukes A, B and C, when patients with distant metastasis (Dukes D), TFU and RTL were striking shorted. This observation was also reported in a previous research that TL was statistically significantly shorter in liver-metastasis tumors than primary tumor and the adjacent non-cancerous liver tissues (20). It has been found that depending on the drug combination, chemotherapy exerted a degree of transient telomere shortening effect and could be recovered to normal TL in around 2 years (32). So the short telomeres in liver metastases tissue were conjectured due to the treatment in CRC, since all the patients with distant metastases underwent various regimens of chemotherapy (20). Our date had a discrepancy between them, because all the patients involved in this research didn't receive any chemotherapy at all. The clinical outcome of different tumor location is varying and prognosis of colon cancer is better than that of rectal cancer (33). Currently, it's under debate whether rectal cancer is actually a distinct entity. In relation to telomere length, it is noteworthy that, although previous results demonstrated that telomere length differed according to tumor location, being longer in rectal cancers $(18,21,29)$, the relationship between telomere length and tumor location was still controversial. We did not find any relationship between telomere length and tumor location just like other studies $(15,16,19,34)$.

We demonstrated a shorted OS and DFS in CRC patients with a decreased TFU and RTL. To minimize the influence of Dukes D stage patients (lower TFU, RTL and high dead rate), lower TFU was still associated with poor OS after excluded them. Some studies had been Long telomere length in cancer tissues as predictors of poorer survival $(10,15,34)$. The reason for aggressive tumor exhibited constantly critically short telomere was speculated as: malignant cells undergoing rapidly cell division required high level of telomerase activity, however, the cellular machinery could not be always guaranteed. The prognostic value of telomere in CRC was discordant in relation to the telomere alterations and prognosis. Some studies found no evidence of cancer RTL associated with disease-free survival or OS, some even opposite to our observation $(16,19-20,21,28,30,35)$. Taken together, the use of telomere as clinical prognostic parameter is still a challenge. Additional mechanism, such as telomere maintenance, telomerase activity alteration and genome instability, should be investigated to completely interpret the different clinical prognosis based on the telomere status.

There are some limitations in this study. First of all, this is a single-center study and the number of patients is relatively larger than most of other studies, but it is nonetheless limited by sample size, particularly as adenoma were further divided into smaller subgroups. Secondly, our survey also has inherent limitations that relate to the applied methods. We can measure TFUs in single adenoma cell and single carcinoma cell, but Q-FISH is not a high-throughput method for large-scale epidemiologic study.

\section{Conclusions}

In conclusion, the present study demonstrated that telomere in carcinoma and adenoma cells were consistently shorter, indicating the telomere changes were early carcinogenesis event, even the epithelial cells were morphologically not malignant. Telomere in carcinoma was associated with tumor metastases and was associated with clinical prognosis.

\section{Acknowledgments}

We thank Dr Xiaosun Liu for his constructive review and English editing of this manuscript.

Funding: None.

\section{Footnote}

Reporting Checklist: The authors have completed the REMARK reporting checklist. Available at https://dx.doi. org/10.21037/tcr-20-3341

Data Sharing Statement: Available at https://dx.doi. org/10.21037/tcr-20-3341

Peer Review File: Available at https://dx.doi.org/10.21037/ tcr-20-3341

Conflicts of Interest: All authors have completed the ICMJE uniform disclosure form (available at https://dx.doi. org/10.21037/tcr-20-3341). The authors have no conflicts of interest to declare.

Ethical Statement: The authors are accountable for all 
aspects of the work in ensuring that questions related to the accuracy or integrity of any part of the work are appropriately investigated and resolved. The study was conducted in accordance with the Declaration of Helsinki (as revised in 2013). The study was approved by the ethics committee of the first affiliated hospital, ZheJiang University School of Medicine (No.2020-656) and informed consent was taken from all the patients.

Open Access Statement: This is an Open Access article distributed in accordance with the Creative Commons Attribution-NonCommercial-NoDerivs 4.0 International License (CC BY-NC-ND 4.0), which permits the noncommercial replication and distribution of the article with the strict proviso that no changes or edits are made and the original work is properly cited (including links to both the formal publication through the relevant DOI and the license). See: https://creativecommons.org/licenses/by-nc-nd/4.0/.

\section{References}

1. Bray F, Ferlay J, Soerjomataram I, et al. Global cancer statistics 2018: GLOBOCAN estimates of incidence and mortality worldwide for 36 cancers in 185 countries CA Cancer J Clin 2018;68:394-424. Erratum in: CA Cancer J Clin 2020;70:313.

2. Ponz de Leon M, Di Gregorio C. Pathology of colorectal cancer. Dig Liver Dis 2001;33:372-88.

3. Markowitz SD and Bertagnolli MM. Molecular origins of cancer: Molecular basis of colorectal cancer. N Engl J Med 2009;361:2449-60.

4. Blackburn EH, Greider CW and Szostak JW. Telomeres and telomerase: the path from maize, Tetrahymena and yeast to human cancer and aging. Nature Med 2006;12:1133-8.

5. Palm $W$ and de Lange $T$. How shelterin protects mammalian telomeres. Annu Rev Genet 2008;42:301-34.

6. Hackett JA, Greider CW. Balancing instability: dual roles for telomerase and telomere dysfunction in tumorigenesis. Oncogene 2002;21:619-26.

7. Meeker AK, Hicks JL, Iacobuzio-Donahue CA, et al. Telomere length abnormalities occur early in the initiation of epithelial carcinogenesis. Clin Cancer Res 2004;10:3317-26.

8. Perera SA, Maser RS, Xia H, et al. Telomere dysfunction promotes genome instability and metastatic potential in a K-ras p53 mouse model of lung cancer. Carcinogenesis 2008;29:747-53.
9. Popat S, Hubner R, Houlston RS. Systematic review of microsatellite instability and colorectal cancer prognosis. J Clin Oncol 2005;23:609-18.

10. Bertorelle R, Rampazzo E, Pucciarelli S, et al. Telomeres, telomerase and colorectal cancer. World J Gastroenterol 2014;20:1940-50.

11. Druliner BR, Ruan X, Johnson R, et al. Time Lapse to Colorectal Cancer: Telomere Dynamics Define the Malignant Potential of Polyps. Clin Transl Gastroenterol 2016;7:e188. Erratum in: Clin Transl Gastroenterol 2017;8:e88.

12. Piñol-Felis C, Fernández-Marcelo T, Viñas-Salas J, et al. Telomeres and telomerase in the clinical management of colorectal cancer. Clin Transl Oncol 2017;19:399-408.

13. Plentz RR, Wiemann SU, Flemming P, et al. Telomere shortening of epithelial cells characterises the adenomacarcinoma transition of human colorectal cancer. Gut 2003;52:1304-7.

14. Giunco S, Rampazzo E, Celeghin A, et al. Telomere and Telomerase in carcinogenesis: Their role as prognostic biomarkers. Curr Pathobiol Rep 2015;3:315-28.

15. Engelhardt M, Drullinsky P, Guillem J, et al. Telomerase and telomere length in the development and progression of premalignant lesions to colorectal cancer. Clin Cancer Res 1997;3:1931-41.

16. Gertler R, Rosenberg R, Stricker D, et al. Telomere length and human telomerase reverse transcriptase expression as markers for progression and prognosis of colorectal carcinoma. J Clin Oncol 2004;22:1807-14.

17. Takagi S, Kinouchi Y, Hiwatashi N, et al. Telomere shortening and the clinicopathologic characteristics of human colorectal carcinomas. Cancer 1999;86:1431-6.

18. Rampazzo E, Bertorelle R, Serra L, et al. Relationship between telomere shortening, genetic instability, and site of tumour origin in colorectal cancers. Br J Cancer 2010;102:1300-5.

19. Valls C, Piñol C, Reñé JM, et al. Telomere length is a prognostic factor for overall survival in colorectal cancer. Colorectal Dis 2011;13:1265-72.

20. Kroupa M, Rachakonda SK, Liska V, et al. Relationship of telomere length in colorectal cancer patients with cancer phenotype and patient prognosis. Br J Cancer 2019;121:344-50.

21. Garcia-Aranda C, de Juan C, Diaz-Lopez A, et al. Correlations of telomere length, telomerase activity, and telomeric-repeat binding factor 1 expression in colorectal carcinoma. Cancer 2006;106:541-51.

22. Tommelein J, Verset L, Boterberg T, et al. Cancer- 
associated fibroblasts connect metastasis-promoting communication in colorectal cancer. Front Oncol 2015;5:63.

23. Paulsson J, Micke P. Prognostic relevance of cancerassociated fibroblasts in human cancer. Seminars in cancer biology 2014;25:61-8.

24. Heaphy CM, Yoon GS, Peskoe SB, et al. Prostate cancer cell telomere length variability and stromal cell telomere length as prognostic markers for metastasis and death. Cancer Discov 2013;3:1130-41.

25. Söreide K, Janssen EA, Söiland H, et al. Microsatellite instability in colorectal cancer. Br J Surg 2006;93:395-406.

26. Fearon ER, Vogelstein B. A genetic model for colorectal tumorigenesis. Cell 1990;61:759-67.

27. Raynaud CM, Jang SJ, Nuciforo P, et al. Telomere shortening is correlated with the DNA damage response and telomeric protein down-regulation in colorectal preneoplastic lesions. Ann Oncol 2008;19:1875-81.

28. Suraweera N, Mouradov D, Li S, et al. Relative telomere lengths in tumor and normal mucosa are related to disease progression and chromosome instability profiles in colorectal cancer. Oncotarget 2016;7:36474-88.

29. Valls Bautista C, Piñol Felis C, Reñé Espinet JM, et al.

Cite this article as: Ye X, Li J, Song C, Chen W. Telomere in colorectal cancer associated with distant metastases and predicted a poor prognosis. Transl Cancer Res 2021;10(6):29062917. doi: 10.21037/tcr-20-3341
Telomerase activity and telomere length in the colorectal polyp-carcinoma sequence. Rev Esp Enferm Dig 2009;101:179-86.

30. Fernández-Marcelo T, Sánchez-Pernaute A, Pascua I, et al. Clinical Relevance of Telomere Status and Telomerase Activity in Colorectal Cancer. PLOS One 2016;11:e0149626.

31. Hao XD, Yang Y, Song X, et al. Correlation of telomere length shortening with TP53 somatic mutations, polymorphisms and allelic loss in breast tumors and esophageal cancer. Oncol Rep 2013;29:226-36.

32. Benitez-Buelga C, Sanchez-Barroso, L, Gallardo, M, et al. Impact of chemotherapy on telomere length in sporadic and familial breast cancer patients. Breast Cancer Res Treat 2015;149:385-94.

33. Li FY, Lai MD. Colorectal cancer, one entity or three. J Zhejiang Univ Sci B 2009;10:219-29.

34. Balc'h EL, Grandin N, Demattei MV, et al. Measurement of Telomere Length in Colorectal Cancers for Improved Molecular Diagnosis. Int J Mol Sci 2017;18:18-71.

35. Jia H, Wang Z. Telomere Length as a Prognostic Factor for Overall Survival in Colorectal Cancer Patients. Cell Physiol Biochem 2016;38:122-8. 